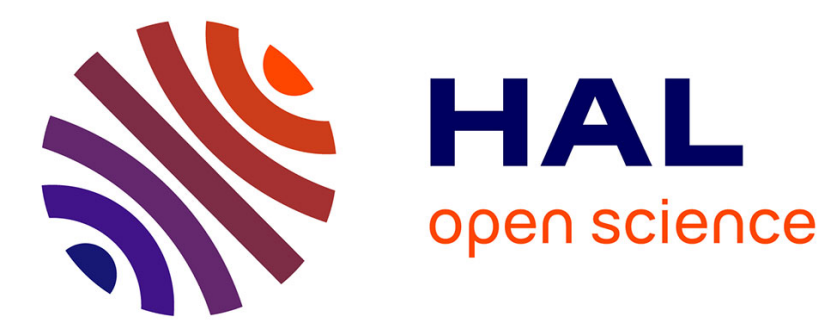

\title{
Reliability assessment of phased-mission systems under random shocks
}

\author{
Xiang-Yu Li, Yan-Feng Li, Hong-Zhong Huang, Enrico Zio
}

\section{To cite this version:}

Xiang-Yu Li, Yan-Feng Li, Hong-Zhong Huang, Enrico Zio. Reliability assessment of phased-mission systems under random shocks. Reliability Engineering and System Safety, 2018, 180, pp.352-361. 10.1016/j.ress.2018.08.002 . hal-01988937

\section{HAL Id: hal-01988937 https://hal.science/hal-01988937}

Submitted on 8 Feb 2019

HAL is a multi-disciplinary open access archive for the deposit and dissemination of scientific research documents, whether they are published or not. The documents may come from teaching and research institutions in France or abroad, or from public or private research centers.
L'archive ouverte pluridisciplinaire HAL, est destinée au dépôt et à la diffusion de documents scientifiques de niveau recherche, publiés ou non, émanant des établissements d'enseignement et de recherche français ou étrangers, des laboratoires publics ou privés. 


\title{
Reliability assessment of phased-mission systems under random shocks
}

\author{
Li Xiang-Yu ${ }^{\mathrm{a}, \mathrm{b}}$, Li Yan-Feng ${ }^{\mathrm{a}, \mathrm{b}}$, Huang Hong-Zhong ${ }^{\mathrm{a}, \mathrm{b}, *}$, Enrico Zio ${ }^{\mathrm{c}, \mathrm{d}}$ \\ a School of Mechanical and Electrical Engineering, University of Electronic Science and Technology of China, Chengdu 611731, China \\ ${ }^{\mathrm{b}}$ Center for System Reliability and Safety, University of Electronic Science and Technology of China, Chengdu 611731, China \\ c Chair System Science and the Energy Challenge, Fondation Électricité de France (EDF), CentraleSupélec, Université Paris Saclay, Gif-sur-Yvette cedex 91192, France \\ d Energy Department, Politecnico di Milano, Milano, Italy
}

\section{A R T I C L E I N F O}

\section{Keywords:}

Phased-mission system

Random shocks

Markov regenerative process

Monte Carlo simulation

Altitude and orbit control system

\begin{abstract}
A B S T R A C T
Phased-mission systems (PMSs) are widely used, especially in the aerospace industry. As in the outer space there are many kinds of cosmic rays, such as the Galactic Cosmic Rays (GCR), randomly hitting on these systems and causing significant impact on the electronics inside or outside the equipment, a reliability model for PMSs considering both finite and infinite random shocks is proposed in this paper. The modularization method is used to simplify the state space model for each phase and reduce the amount of system states, and the Markov regenerative process (MRGP) is used to describe the hybrid components' lifetime distributions and the dynamic behaviors within the modules. Then, two kinds of scenarios, finite and infinite random shocks effect, are both integrated into the dynamic modules. For demonstration, a phased altitude and orbit control system (AOCS) subjected to infinite random shocks is illustrated to demonstrate the procedure of the proposed Monte Carlo simulation. Thirdly, the evaluated system reliability under infinite random shocks is compared with the same system without considering random shocks. At last, a sensitivity analysis is also provided for completion.
\end{abstract}

\section{Introduction}

In this paper, the reliability of phased-mission systems (PMSs) subjected to random shocks is considered. In a PMS, the system needs to perform different tasks in successive time periods, known as phases [1]. A classic example is the manned spacecraft whose missions can be divided into launch, orbit-transfer, on-orbit operation and back-to-earth phases. In these non-overlapping phases, the system needs to accomplish different mission demands. For these complex and high-value aerospace equipment, reliability is a very critical value. Usually, the reliability of PMSs is defined as the probability that all the consecutive missions are accomplished successfully. The challenges in the reliability assessment of PMSs are mainly due to three aspects: (1) dynamic behaviors within phases, like the CSP (cold spare) which is commonly used to improve the system reliability or the FDEP (functional DEPendent); (2) dynamic behaviors among phases, whereby the system configuration changes from one phase to another; moreover, in different phases the system will be subjected to different environments, which may lead to different stresses and failure rates [1,2]; (3) phase dependence, in which the components failed in the former phases will remain failed in the later phases, in non-repairable PMSs [3].

The existing works on the reliability modeling of PMSs can be classified into two major categories:
(1) Combinatorial methods, like binary decision diagram (BDD) [1-6] or multi-valued decision diagram (MDD) based models [7]. A BDD is a direct acyclic graph that is based on Shannon decomposition and the graph has two sink nodes, labeled 0 and 1 , representing the system working or failure [2]. MDD models are natural extension of traditional BDD models which has multiple outgoing edges to represent the system being in different states [7] that are commonly used in multistate system or system with multiple failure modes. The BDD method was applied by Zang and Trivedi [2] to assess the system reliability of a PMS. Xing applied the BDD method in the reliability analysis of a generalized PMS, considering the phase-OR as a special case of PMS [3]. Xing also used the BDD method to assess the system reliability of PMS considering both common cause failure (CCF) and imperfect coverage [1], and the PMS considering both internal/external CCF [5]. Tang and Dugan assessed the system reliability of PMS considering multimode failures by the BDD method [6]. Besides the BDD modeling method, the MDD modeling method has also been applied for PMS reliability analysis, especially considering multi-failure modes. Mo [7] pointed out that MDD modeling method is more efficient than BDD method in PMS with multi-failure modes. In general, combinatorial methods can assess the system reliability efficiently, especially in large scale systems. But they can only deal with the static system that all the primary events in the phase fault tree (FT) model are independent on

\footnotetext{
* Corresponding author at: School of Mechanical and Electrical Engineering, University of Electronic Science and Technology of China, Chengdu 611731, China.

E-mail address: hzhuang@uestc.edu.cn (H.-Z. Huang).
} 


\begin{tabular}{|c|c|c|c|}
\hline \multicolumn{2}{|c|}{ Nomenclature } & BDD & Binary decision diagram \\
\hline Mi & The ith module after system modularization & CSP & Cold spare \\
\hline$M$ & The states set of the modules & FDEP & Functional DEPendent \\
\hline$T_{n}$ & The phase time of phase $n$ & FT & Fault tree \\
\hline$n$ & The phase number & GCR & Galactic cosmic ray \\
\hline$u$ & The arrival rate of the random shocks & MC & Monte Carlo \\
\hline$\lambda_{S}$ & The failure rate of component $S$ without shocks & MDD & Multi-valued decision diagram \\
\hline$\lambda_{S}^{n}$ & The failure rate of component $S$ after the $n$ thshocks & MRGP & Markov regenerative process \\
\hline$T_{F}$ & The recorded module failure time & PMS & Phased-mission system \\
\hline$R_{\text {sys }}(t)$ & The system reliability at time $t$ & SSE & Single event effects \\
\hline
\end{tabular}

Acronym

AOCS Altitude and orbit control system

each other.

(2) State space oriented models, like Markov chain-based or Petri net-based models [8-10]. In the state space oriented models, the dynamic behaviors in each phase are represented by state space models, Markov chains or Petri nets. Then the phase dependence is involved in if components' states do not change during the jump of the phases. These models can deal with the dynamic behaviors within phases, like the CSP, but they suffer from the state explosion problem, especially in the large scale system.

To overcome the disadvantages of the methods above, a modularization method combined with combinatorial and state space models is proposed in Refs. [11,12]. Through the modularization method, the dynamic components are separated as some into independent modules. As a result, the system can be evaluated by the combinatorial methods and the independent modules. Therefore, the modularization method combines the advantages of both methods.

The PMS considered in this paper is employed in the aerospace industry, e.g., the manned spacecraft. These systems spend most of their lifetime in the outer space, where they are exposed to many kinds of cosmic rays, such as the galactic cosmic rays (GCRs) [13]. The ionizing nature of GCR particles can pose significant threats to the electronics located onboard, such as the microprocessors to which they may cause memory bit flips and latch-ups. This kind of phenomenon is generally called the single event effect (SSE) [13] and occurs randomly, i.e. as a random shock. If these random shocks are not considered, the reliability of the PMS will be overestimated.

Random shocks have been considered with different approaches in reliability modeling [14-24]. Lin and Zio [14] studied the components' reliability considering both degradation processes and random shocks. At the system level, Wang and Pham [17] investigated the influence of the degradation and random shocks, in which the random shocks can lead the system to failure immediately. Rafiee [18] studied cumulative random shocks that increase the components' failure rates. Berker [19] used a semi-Markov model to describe a system under random shocks. Recently, Ruiz-Castro [20] considered the extreme failures and cumulative damage caused by the external shocks. However, these methods are all considered in single-phased systems.

The main contribution of this paper is to integrate random shocks into the reliability modeling of PMSs and a Monte Carlo simulation procedure is then developed for its quantification [26-28]. Firstly, the modularization method is used to divide the system into several individual modules so that the complicated system FT model can be simplified. Secondly, the random shocks are integrated into the state space model of the modules by the MRGP. Thirdly, a Monte Carlo method for assessing module reliabilities under random shocks is developed. Finally, the reliability of the PMS is evaluated through the PMS-BDD method and the mutually independent modules.

The paper is organized as follows. In Section 2, the basic conceptions of the Markov regenerative process and an altitude and orbit control system (AOCS) of the manned spacecraft are introduced in detail. To model the reliability efficiently, the modularization method is applied to simplify its system FT model. Then, the dynamic module is modeled by the MRGP. In Section 3, the model for the dynamic module under random shocks and the MC simulation procedure for assessing the reliability of the dynamic module under infinite random shocks are proposed. After that, a dynamic module under finite random shocks is evaluated by the MC simulation and approximation method, respectively, and the reliability comparison result certifies the proposed MC simulation procedure. In Section 4, the reliability of the AOCS under infinite random shocks is evaluated by integrating the PMS-BDD method and evaluated module reliabilities in previous. Furthermore, a comparison between the system reliability analysis with and without random shocks is also provided. Furthermore, the confidence of the MC simulation method and a sensitivity analysis is performed. The modeling procedure is shown in Fig. 1. The summary of the work and main conclusions are presented in Section 5.

\section{MRGP and multi-phased AOCS}

\subsection{The multi-phased AOCS}

In this paper, the reliability of the AOCS in a manned spacecraft under random shocks is studied. The AOCS (altitude and orbit control system) is a critical subsystem of the manned spacecraft to control and adjust its altitude and orbit in the whole lifetime. If the AOCS fails, the manned spacecraft cannot stay in the right altitude and orbit.

\subsubsection{System working procedure of AOCS}

The AOCS consists of three functional subsystems-the sensors, the processors and the actuators. The working procedure of the AOCS is shown in Fig. 2. Firstly, the sensors acquire the altitude and orbit data

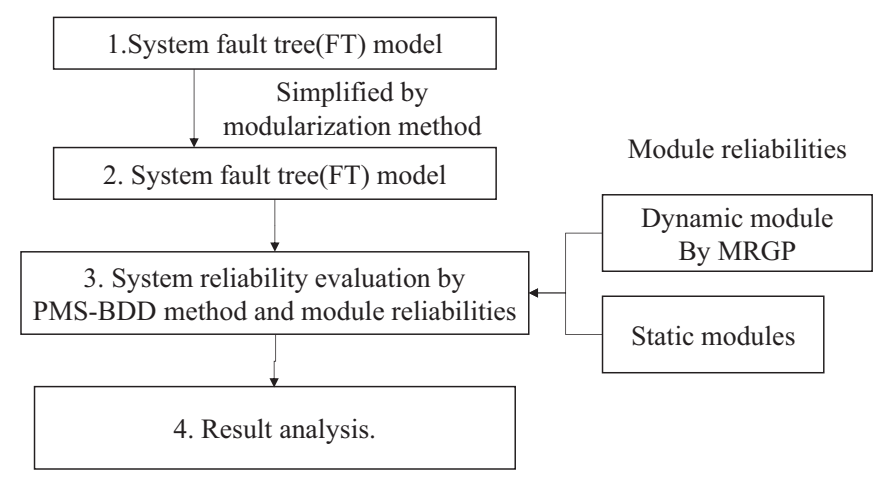

Fig. 1. The system reliability evaluation procedure. 


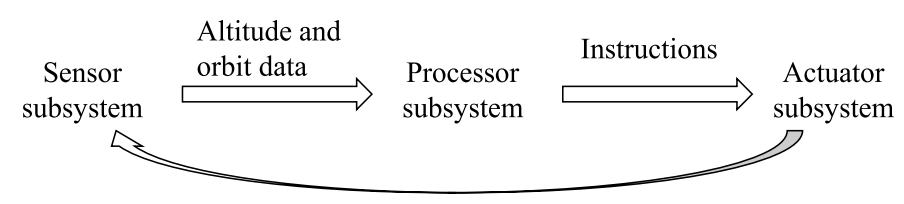

Fig. 2. The working procedure of the AOCS.

and send it to the processors. Secondly, the processors process the data and make decisions, and then, the instructions are sent to the actuators. Finally, the actuators adjust the altitude and orbit according to the instructions. The repetition of this working procedure will keep the spacecraft on the right altitude and orbit during its whole lifetime.

Considering that different missions need to be accomplished at different time, the whole lifetime of the AOCS can be divided into four phases: launching phase, orbital-transfer phase, on-orbit phase and back-to-earth phase. The phase durations of four phases are $T_{1}=2$ days, $T_{2}=4$ days, $T_{3}=30$ days and $T_{4}=3$ days, shown in Fig. 3 .

\subsubsection{Components and system structure}

As described above, the AOCS is composed of three functional parts;

(1) Micro-computers (Processors): working computer (A), standby computer $(B)$ and a switch component $(C)$. (2) Sensors: sun sensor $(D)$, earth sensor $(E)$, star track sensor $(F)$ and gyro assembly $(G)$. (3) Actuators: low thrust thrusters $(20 \mathrm{~N}$, cold standby subsystem, working thruster $H$, cold standby thruster $I$ and a switch component $S$ ), high thrust thruster $(620 \mathrm{~N}, Q$ ), three Momentum wheels (2 out of 3 subsystem, $J, K$ and $L$ ).

All the components can be divided into two categories: The working and standby components and the switch components. The lifetime of the complicated working components follow the Weibull distributions $\left(F(t)=1-e^{-(t / \alpha)^{\beta}}\right.$, where $\alpha$ is the shape parameter and $\beta$ is the scale parameter). And the lifetime of the switches (electronics) follow the exponential distributions $\left(F(t)=1-e^{-\lambda t}\right.$, where $\lambda$ is the failure rate). Due to the confidential requirement, the original data are unavailable, and the parameters of the components are provided by the designers of the spacecraft after being processed, as shown in Table 1 .

In the launching phase (1st phase), the spacecraft is launched into the outer space and separated from the rocket. In this phase, the sensors and the processors are necessary to acquire the position data and process it. The processers $(A, B$ and $C$ ) and the sensors $(D, E, F$ and $G)$ are necessary. The FT model of phase 1 is shown as Fig. 4(a).

In the orbit-transfer phase (2nd phase), the spacecraft needs to be transferred to the working orbit step by step. Besides the processors and the sensors, the high-thrust thruster 2 and low-thrust thruster 1 are used for the orbit transfer and orbit micro-adjusting, respectively. In this phase, the processers $(A, B$ and $C$ ), the sensors $(D, E, F$ and $G)$, the highthrust thruster $(Q)$ and the low-thrust thruster $(H, I$ and $S$ ) are necessary. The FT model of phase 2 is shown as Fig. 4(b).

In the on-orbit phase (3rd phase), the spacecraft works in the normal orbit and the AOCS needs to keep the spacecraft in the correct altitude and orbit. In this phase, except for the micro-computers and sensors, the thruster 1 and momentum wheels are used as actuators to keep the spacecraft working normally on the right orbit. The processers $(A, B$ and $C$ ), the sensors ( $D, E, F$ and $G$ ), the low-thrust thruster ( $H, I$ and $S$ ) and the movement wheels $(J, K$ and $L)$ are necessary and the FT model of phase 3 is shown as Fig. 4(c).

In the back-to-earth phase (4th phase). The spacecraft need to transfer to the lower orbit and then return to earth. In this phase, the processers $(A, B$ and $C$ ), the sensors $(D, E, F$ and $G)$ and the low-thrust thruster $(H, I$ and $S$ ) are necessary and the FT model of phase 4 is shown in Fig. 4(d).

\subsection{Simplified by modularization method}

In the previous section, the mission profile and FT models of different phases have been described. Directly applying state-space modeling methods in system modeling for each phase would lead to a very large number of states for each phase, known as the state explosion problem [10], which would make it very difficult to evaluate the system model. In this paper, the modularization method is used to simplify the system FT models and the state explosion problem can be solved to some extent.

The modularization method is proposed by Khoda [29] and used in reliability assessment of PMS by Ou and Dugan [11]. A phase module of a multi-phased system must meet two conditions [11]: (1) each module is a set of the basic events, which means a module must be a subset of all basic events; (2) for each phase, the basic events in the collection should form an independent sub-tree in the modularized fault tree. According to these conditions, all the bottom events in the FT models in Fig. 4 of the ACOS can be divided into five independent modules: $M 1=(A, B, C), M 2=(D, E, F, G), M 3=(H, I, S),, M 5=(J, K, L)$, as shown in Fig. 5(a)-(e), respectively.

With these modules, the FT model for the entire multi-phased AOCS after modularization is shown in Fig. 6. All the modules can be regarded

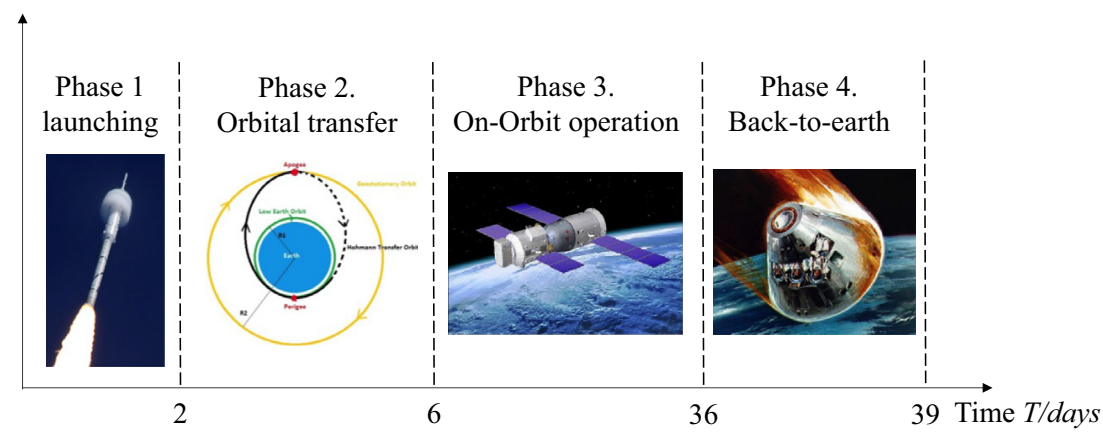

Fig. 3. The mission profile for the AOCS.

Table 1

The parameters for the phased AOCS.

\begin{tabular}{|c|c|c|c|c|c|c|c|}
\hline & $A$ & $B$ & $D / E / F / G$ & $H$ & $I$ & $Q$ & $J / K / L$ \\
\hline$\alpha$ & 1.563 & 1.725 & 2.156 & 2.093 & 2.185 & 1.937 & 1.358 \\
\hline \multirow[t]{2}{*}{$\beta$ (days) } & 138.12 & 172.65 & 1291.50 & 133.15 & 188.72 & 332.84 & 287.47 \\
\hline & C & $S$ & & & & & \\
\hline$\lambda$ (days $^{-1}$ ) & $1 / 201.95$ & $1 / 216.01$ & & & & & \\
\hline
\end{tabular}




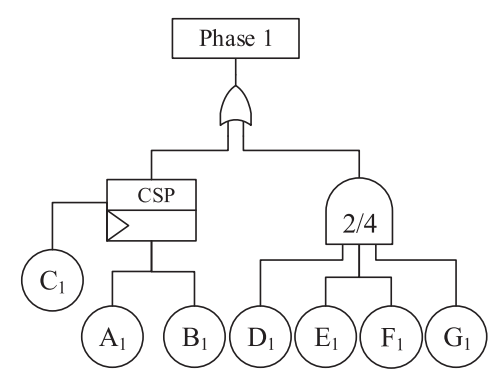

(a)

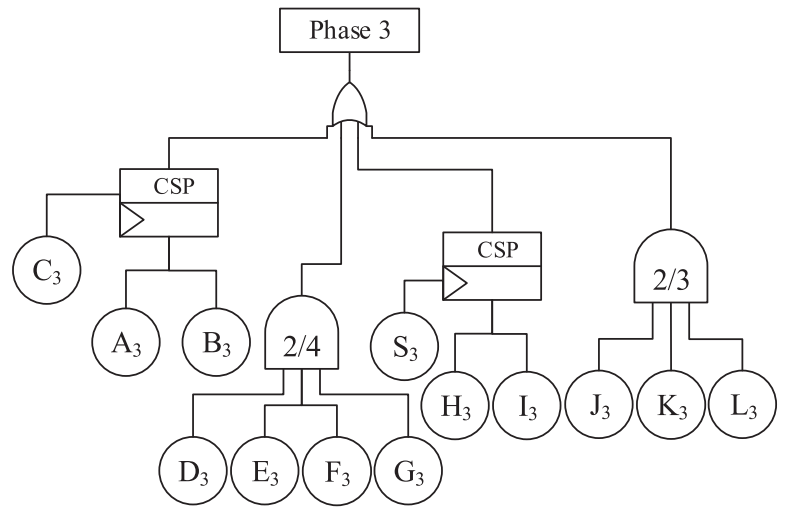

(c)

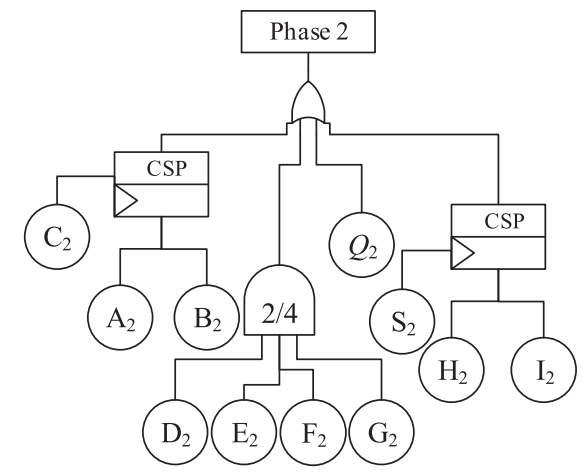

(b)

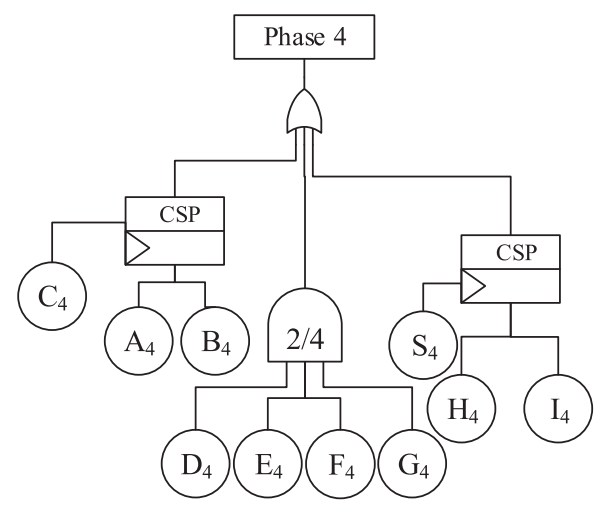

(d)

Fig. 4. The FT models for each phase of the AOCS.

as independent bottom events in the modularized FT model and the system reliability can be assessed by the PMS-BDD method [2] and the module reliabilities. If there are any dynamic logic gates, the modules are dynamic modules [30]. The reliability of the static modules can be easily evaluated by their own RBD. Moreover, the reliability of the dynamic modules will be evaluated by the Markov regenerative process (MRGP).

\subsection{The MRGP model for the dynamic module}

\subsubsection{Basic conceptions of MRGP}

In general, a Markov generative process $\{Y(t), t \geq 0\}$ does not possess the Markov property (the memoryless property). But there is a sequence of embedded time sequence within $Y(t)$, the Embedded Markov chain (EMC) $(X, S)=\left\{X_{n}, S_{n}\right\}$, which is also called the Markov regenerative sequence (MRS). It satisfies the Markov property in the Markov regenerative time points [25],

$$
\begin{gathered}
\operatorname{Pr}\left\{X_{n+1}=j, S_{n+1}-S_{n} \leq t \mid X_{n}=i, \cdots \cdots x_{0} ; S_{n}, \cdots \cdots, S_{0}\right\} \\
=\operatorname{Pr}\left\{X_{n+1}=j, S_{n+1}-S_{n} \leq t \mid X_{n}=i\right\}
\end{gathered}
$$

where $X_{n}$ and $S_{n}$ are the state being visited and the $n$th transition time.

$S_{n}$ in Eq. (1) is the Markov regenerative points and the stochastic process $Y(t)$ possesses the Markov property at these time points. With the embedded MRS $(X, S)$, the MRGP $Y(t)$ satisfies,

$\operatorname{Pr}\left\{Y_{t+S_{n}}=j \mid Y_{u}, 0 \leq u \leq S_{n}, X_{n}=i\right\}=\operatorname{Pr}\left(Y_{t}=j \mid X_{0}=i\right)$.

According to Eq. (2), it can be found that the future of $Y(t)$ from $t=S_{n}$ only depends on the past only through state $X_{n}$. To define a Markov regenerative process, the conditional probability matrix $\boldsymbol{\theta}(t)$ is defined as,

$\theta_{i, j}(t)=\operatorname{Pr}(Y(t)=j \mid Y(0)=i)$.

In majority of reliability problems involving the MRGP, the primary concern is to evaluate the conditional probability matrix $\boldsymbol{\theta}(t)$. In the evaluation of $\boldsymbol{\theta}(t)$, two matrices, the $\boldsymbol{Q}(t)$ and $\boldsymbol{E}(t)$, are necessary.

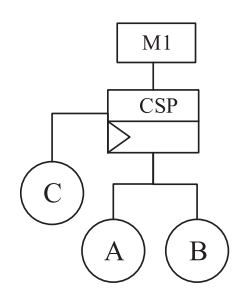

(a)

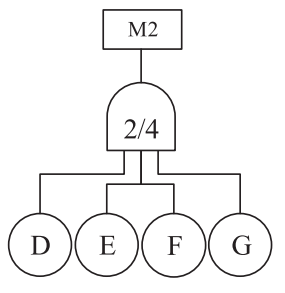

(b)

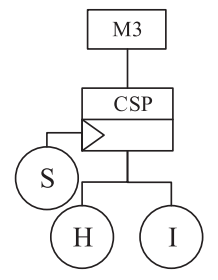

(c)

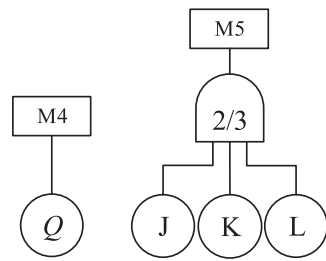

(d)

(e)

Fig. 5. The modules for the AOCS. 


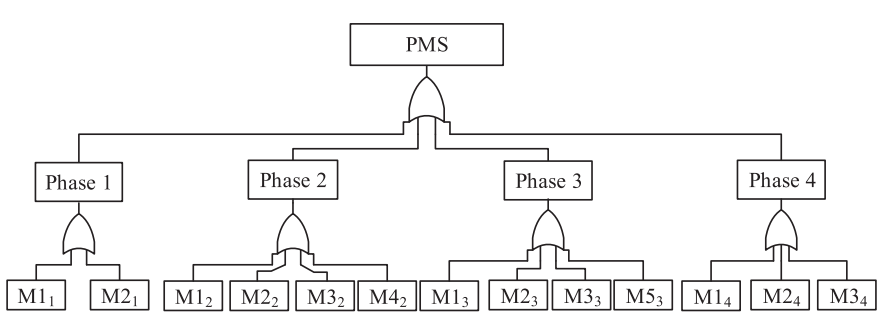

Fig. 6. The modularized fault tree of the multi-phased AOCS.

$\boldsymbol{Q}(t)=\left[Q_{i, j}(t)\right]$ and $\boldsymbol{E}(t)=\left[E_{i, j}(t)\right]$ are called the global kernel and the local kernel of the MRGP, respectively, which are defined as,

$Q_{i, j}(t)=\operatorname{Pr}\left\{Y(t)=j, S_{1} \leq t \mid Y(0)=i\right\}$

$E_{i, j}(t)=\operatorname{Pr}\left\{Y(t)=j, S_{1}>t \mid Y(0)=i\right\}$

From Eq. (4), the local kernel, $E_{i, j}(t)$, describes the state transition behavior of the MRGP during two consecutive Markov regenerative epochs $\left(0, S_{1}\right)$ and the global kernel, $Q_{i, j}(t)$, describes the state transition behavior immediately after the next Markov regenerative epoch $S_{1}$. With the global kernel $\boldsymbol{Q}(t)$ and the local kernel $\boldsymbol{E}(t)$, the system state transition probability $\boldsymbol{\theta}(t)$ can be evaluated by the Markov renewal equation [25],

$\theta(t)=\boldsymbol{E}(t)+\int_{0}^{t} \theta(t-u) d \boldsymbol{Q}(t)$.

\subsubsection{Dynamic module evaluation by MRGP}

Using the dynamic module $M 3$ is used as an example, the system state evaluation procedure is illustrated as follows:

Step 1: construct the state transition diagram. The state transition diagram of the dynamic module $M 3$ is shown in Fig. 7.

Step 2: identify the structure of the global kernel matrix $\boldsymbol{Q}_{M 3}(t)$ and the local kernel matrix $\boldsymbol{E}_{M 3}(t)$. According to Fig. 7, the exponential transition from state $S 1$ to $S 3$ and from state $S 2$ to $S 4$ are concurrent. It is because that the failure of component $S$ does not affect the failure process of component $H$ and $I$. So states $S 3$ and $S 4$ are not the Markov regenerative epoch. Hence, this stochastic process shown in Fig. 7 is a MRGP whose EMC is identified by states $S 1, S 2$ and $S 5$. Accordingly, the global kernel matrix $\boldsymbol{Q}_{M 3}(t)$ and the local kernel matrix $\boldsymbol{E}_{M 3}(t)$ of this MRGP is,

$\boldsymbol{Q}_{M 3}(t)=\left[\begin{array}{ccccc}0 & Q_{1,2}^{M 3}(t) & 0 & 0 & Q_{1,5}^{M 3}(t) \\ 0 & 0 & 0 & 0 & Q_{2,5}^{M 3}(t) \\ 0 & 0 & 0 & 0 & 0 \\ 0 & 0 & 0 & 0 & 0 \\ 0 & 0 & 0 & 0 & 0\end{array}\right]$

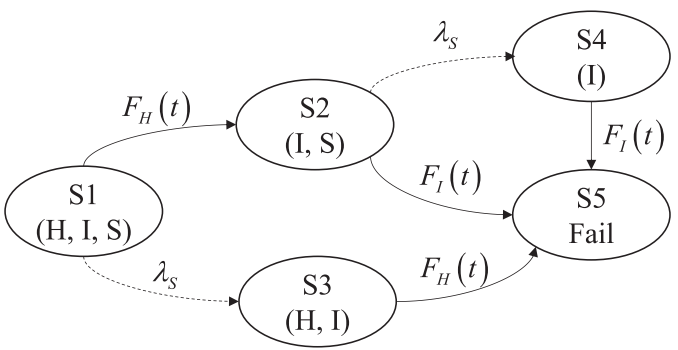

Fig. 7. The state transition diagram for module M3.

$$
\boldsymbol{E}_{M 3}(t)=\left[\begin{array}{ccccc}
E_{1,1}^{M 3}(t) & 0 & E_{1,3}^{M 3}(t) & 0 & 0 \\
0 & E_{2,2}^{M 3}(t) & 0 & E_{2,4}^{M 3}(t) & 0 \\
0 & 0 & 0 & 0 & 0 \\
0 & 0 & 0 & 0 & 0 \\
0 & 0 & 0 & 0 & E_{5,5}^{M 3}(t)
\end{array}\right]
$$

Step 3: evaluate all the elements in $\boldsymbol{Q}_{M 3}(t)$ and $\boldsymbol{E}_{M 3}(t)$. The elements in $\boldsymbol{Q}_{M 3}(t)$ and $\boldsymbol{E}_{M 3}(t)$ can be computed according to the competing failure mechanism. For example, let $T_{H}, T_{I}$ and $T_{S}$ represent the time to failure of components $H, I$ and $S$, respectively. Then,

$$
\begin{aligned}
& Q_{1,2}^{M 3}(t)= \operatorname{Pr}\left\{Y\left(S_{1}\right)=2, S_{1} \leq t \mid Y(0)=1\right\} \\
&= \operatorname{Pr}\left\{{ }^{\prime} \text { Componet } H \text { failsbeforecomponent } S\right. \\
&\text { fails' }\} \\
&=\operatorname{Pr}\left\{T_{H} \leq t \& T_{S}>T_{H}\right\} \\
&=\int_{0}^{t} \operatorname{Pr}\left\{T_{S}>u\right\} d F_{H}(u) \\
&=\int_{0}^{t} f_{H}(u)\left(1-F_{S}(u)\right) d u
\end{aligned}
$$

It should be noted that during the calculation of $Q_{1,2}^{M 3}(t)$, the failure of the component $S$ does not have any effect on the component I. $Q_{2,5}^{M 3}(t)$ can be evaluated as,

$$
\begin{aligned}
Q_{2,5}^{M 3}(t) & =\operatorname{Pr}\left\{\begin{array}{c}
\text { 'Componet } I \text { fails before component } S \text { fails' } \\
\text { or 'Componet } I \text { fails after component } S \text { fails' }
\end{array}\right\} \\
& =\operatorname{Pr}\left\{T_{I} \leq t \& T_{S}>T_{I}\right\}+\operatorname{Pr}\left\{T_{I} \leq t \& T_{S} \leq T_{I}\right\}=\operatorname{Pr}\left\{T_{I} \leq t\right\} \\
& =F_{I}(t)
\end{aligned}
$$

Other elements in the global kernel $\boldsymbol{Q}_{M 3}(t)$ and the local kernel $\boldsymbol{Q}_{M 3}(t)$ can be evaluated in the same way.

Step 4: using the evaluated $\boldsymbol{Q}_{M 3}(t)$ and $\boldsymbol{E}_{M 3}(t)$, the system state transition probability matrix $\boldsymbol{\theta}_{M 3}(t)$ can be evaluated by the Markov renewal equation shown in Eq. (5).

\section{Integrating of random shocks into the PMS reliabiltiy model}

In this section, the random shocks (e.g. coming from cosmic rays) are integrated into the PMS reliability model. After modularization, the system MFT model is shown in the last section and all the bottom events in the FT models have been divided into several independent modules. The dynamic module, cold standby module $M 3$, is used as an example to describe the model considering random shocks. In module $M 3, H$ and $I$ are working components and $S$ is the switch component. According to the module description, the state transition diagram of $M 3$ is shown in Fig. 7 . State $S 1$ is the perfect working state and $S 5$ is the failure state.

As described in the section of Introduction, the randomly coming cosmic rays affect the electronics as random shocks. To integrate the random shocks in the PMS reliability model, some preliminary assumptions are made:

- The arrivals of the random shocks follow a homogeneous Poisson process [14], with a constant arrival rate $u$ (shown in Fig. 8). By the opinion of the spacecraft designers, the random shocks occurrence rate is set to be 5 days once and $u=1 / 5$ days $^{-1}$ in this paper.

- The random shocks and the components' failure process are 


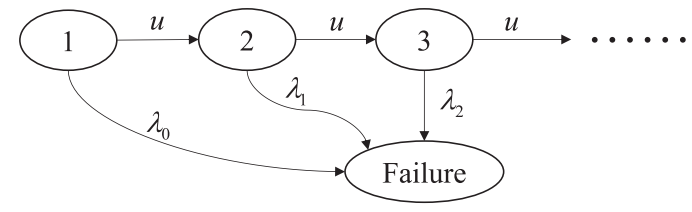

Fig. 8. The infinite random shocks process.

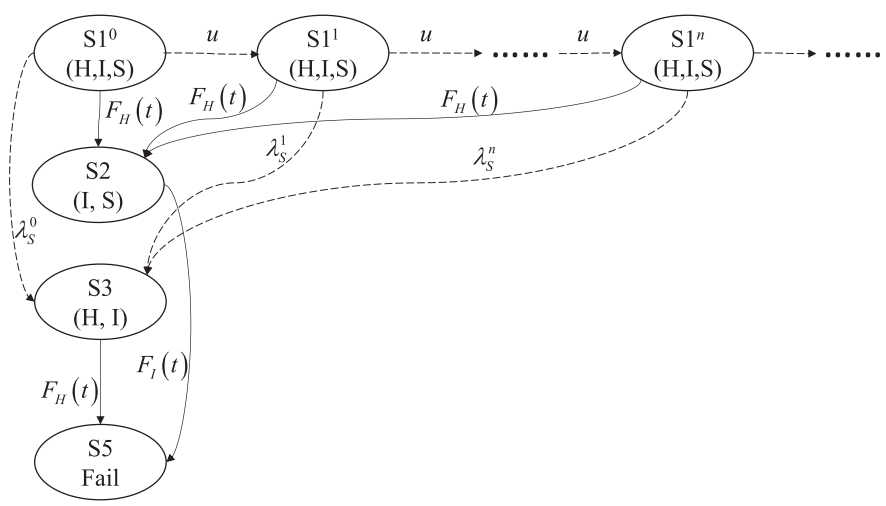

Fig. 9. The state transition diagram under random shocks for module $M 3$.

independent on each other.

- The damage brought by the random shocks is cumulative, and in particular, the random shocks increase the failure rate of a constant amount $\varepsilon$ at each time they occur and cannot lead the components to failure directly.

In this paper, we assume that $M$ indicates the system state and $N$ indicates the number of random shocks that have occurred. To integrate the random shocks in the PMS reliability model, the system state indicator is extended from $M$ into $(M, N)$. After integrating the random shocks as shown in Fig. 8 into the state transition diagram of Fig. 7, the state transition diagram with random shocks for module $M 3$ is shown in Fig. 9.

Furthermore, the failure rates after $n$ random shocks $\lambda_{S}^{n}$ in Fig. 9 are set to be $\lambda_{S}^{n}=\lambda_{S}(1+\varepsilon)^{n}[14]$, where $\lambda_{S}$ represents the transition rate of the system from state $i$ to state $j$ without random shocks and $(1+\varepsilon)^{n}$ characterizes the cumulative effects of the random shocks. By the opinion of experts, the failure rate increment $\varepsilon$, due to a shock, is set to be 0.3 in the case study of this paper. Due to the infinite state number of dynamic module under infinite random shocks, the Monte Carlo (MC) simulation method $[26,27]$ is applied to assess the PMS reliability in this paper. The MC simulation for evaluating the PMS reliability under random shocks.

\subsection{The simulation procedure for dynamic modules}

The MC simulation method for the reliability assessment is based on repeated sampling of realizations of system state configurations and computation of the system failure frequency [31,32]. In this paper, the simulation procedure is conducted to evaluate the reliability of the dynamic module. During each simulation, two quantities, i.e. the number of random shocks $N$ and the module failure time $T_{F}$, are recorded. In each repeated simulation, the failure time of the dynamic module, $T_{F, M_{i}}$, is simulated by the sampled components' failure time and the logic within the dynamic module. Using the module $M 3$ as an example, the simulation procedure for the dynamic modules is shown in Fig. 10.

\subsection{Certification of the MC simulation}

In this section, to certify the proposed MC simulation procedure, the reliability of module $M 3$ under finite random shocks by the proposed MC simulation method is compared to the reliability of the same system by the MRGP and computed by an approximation method.

Considering a scenario that each random shock on component $S$ in module 3 does not only lead to the increasing of its failure rate, but also has a cumulative shock damage and the component will fail after a certain number of random shocks because the cumulative damage reaches the failure threshold $[17,31]$. If component $S$ fails after the 3rd shocks occur, the state transition diagrams for component $S$ under finite random shocks and module 3 under finite random shocks are shown in Figs. 11 and 12, respectively.

According to the system description in Section 2.3, the unreliability of module $M 3$ at time $t$ is the system state transition probability $\theta_{1,5}^{M 3}(t)$. By the Markov renewal equation shown in Eq. (6), the state transition probability $\theta_{1,5}^{M 3}(t)$ can be evaluated as,

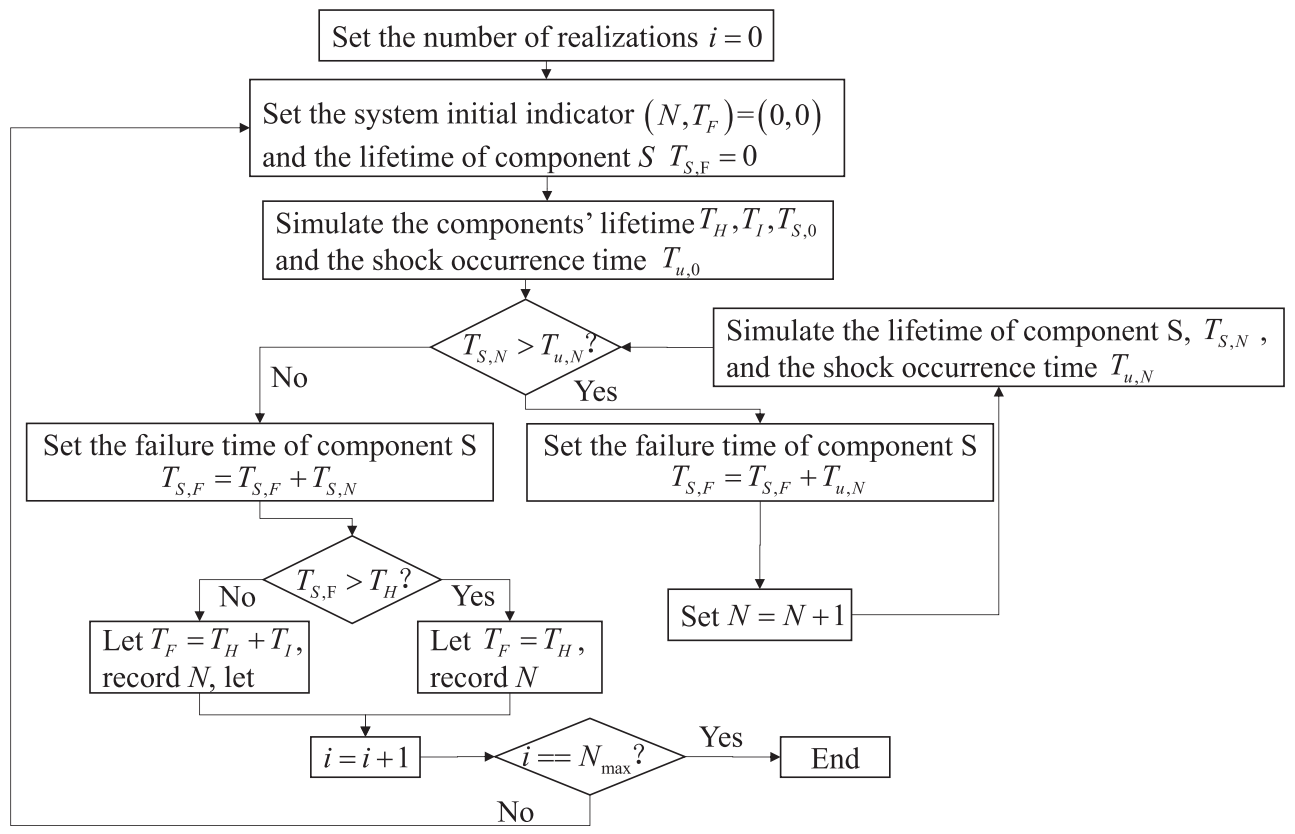

Fig. 10. The simulation procedure for dynamic module under infinite shocks. 


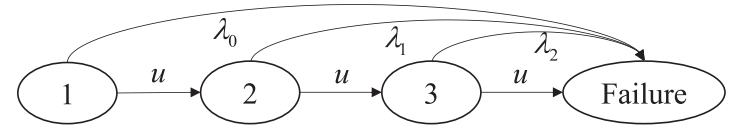

Fig. 11. The state transition diagram for component $S$ under finite shocks.

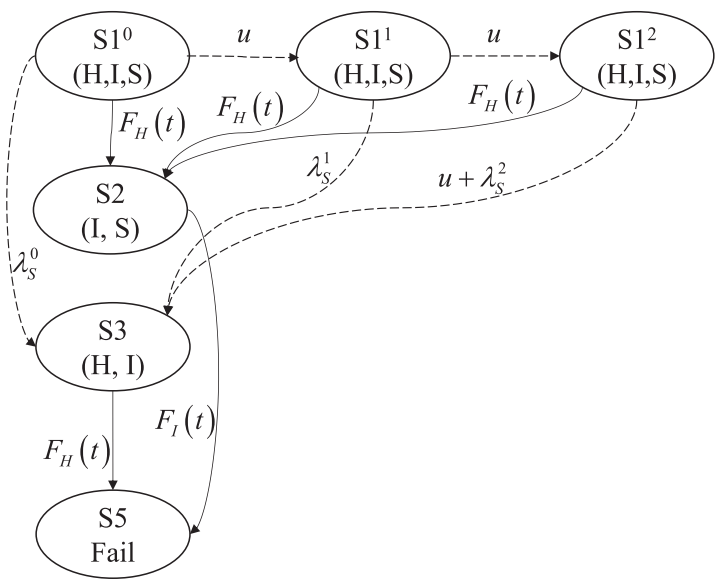

Fig. 12. The state transition diagram for $M 3$ under finite shocks.

$\theta_{1,5}^{M 3}(t)=\int_{0}^{t} q_{1,5}^{M 3}(u) \theta_{5,5}^{M 3}(t-u) d u+\int_{0}^{t} q_{1,2}^{M 3}(u) \theta_{2,5}^{M 3}(t-u) d u$

where $q_{i, j}^{M 3}(t)=d Q_{i, j}^{M 3}(t) / d t$.

In Eq. (10), $\theta_{5,5}^{M 3}(t)=1$ and $\theta_{2,5}^{M 3}(t)=F_{I}(t)$. Then, the $q_{1,5}^{M 3}(t)$ and $q_{1,2}^{M 3}(t)$ can be computed as,

$q_{1,5}^{M 3}(t)=F_{S}(t) d F_{H}(t)$

$q_{1,2}^{M 3}(t)=\left(1-F_{S}(t)\right) d F_{H}(t)$

where $F_{H}(t)$ is the CDF of component $H\left(F_{H}(t)=1-\exp \left(-\left(t / \alpha_{H}\right)^{\beta_{H}}\right)\right)$. $F_{S}(t)$ is the CDF of component $S$ under finite random shocks and can be evaluated by the CTMC [10],

$$
\begin{aligned}
& F_{S}(t)=1+\frac{\left(u\left(\lambda_{2}-\lambda_{S}+u\right)+\lambda_{S}\left(\lambda_{S}-\lambda_{1}-\lambda_{2}\right)+\lambda_{1} \lambda_{2}\right)}{\left(\lambda_{S}-\lambda_{1}\right)\left(\lambda_{S}-\lambda_{2}\right)} \\
&- \exp \left(-\left(\lambda_{S}+u\right) t\right) \\
&-\frac{u^{2}}{\left(\lambda_{S}-\lambda_{2}\right)\left(\lambda_{1}-\lambda_{2}\right)} \exp \left(-\left(\lambda_{2}+u\right) t\right) \\
&-\frac{\left(\lambda_{2} u-\lambda_{1} u+u^{2}\right)}{\left(\lambda_{S}-\lambda_{1}\right)\left(\lambda_{1}-\lambda_{2}\right)} \exp \left(-\left(\lambda_{1}+u\right) t\right)
\end{aligned}
$$

where $u$ is the arriving rate of the random shocks and $\lambda_{i}$ is the failure rate of component $S$ after the $i$ th shocks $\lambda_{i}=\lambda_{S}(1+\varepsilon)^{i}$.

To compute the complicated integrals in Eq. (10), an accurate approximation method, the trapezoidal integration method [33, 34], is applied in this paper and shown as,

$$
\begin{aligned}
\int_{0}^{t} q_{i, k}(\tau) \theta_{k, j}(t-\tau) d \tau & \approx \frac{1}{2} \sum_{i=1}^{n}\left[q_{i, k}\left(\tau_{i}\right) \theta_{k, j}\left(t-\tau_{t}\right)\right. \\
& \left.+q_{i, k}\left(\tau_{i+1}\right) \theta_{k, j}\left(t-\tau_{i+1}\right)\right]\left[\tau_{i+1}-\tau_{i}\right]
\end{aligned}
$$

where the integration interval $[0, t]$ is divided into $n$ equal segments, so the length of each segment is $\delta=t / n$. Integrating Eq. (10)-Eq. (13) into Eq. (10), the system state transition probability $\theta_{1,5}^{M 3}(t)$ can be evaluated. Then, the comparison between the reliabilities of module $M 3$ by the proposed MC simulation procedure and the MRGP as well as the approximation method is shown in Fig. 13.

The results in Fig. 13 demonstrate that the proposed MC simulation

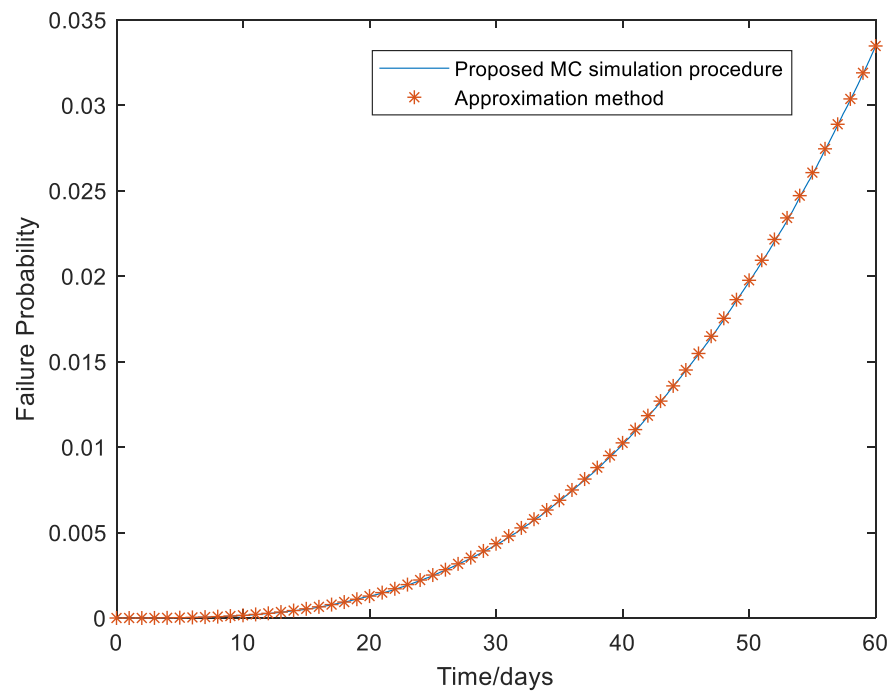

Fig. 13. The comparison of module $M 3$ under finite random shocks by different methods.

procedure for the module under random shocks can provide a relatively accurate result.

\section{Reliability assessment analysis}

\subsection{System reliability assessment by PMS-BDD model}

In Section 2.2, the complex FT model of the AOCS is simplified as the modularized FT model and all the bottom events of the modularized FT are independent on each other. Then, the system reliability can be evaluated by the widely used PMS-BDD model. Considering the phase dependence by the phase algebra proposed in Ref. [6], the system reliability of the phased AOCS can be evaluated by the PMS-BDD model by several steps that are described as follows:

Step 1: transit the FT model of each phase into the corresponding BDD model of each phase. The BDD models for the four phases of the AOCS are shown in Fig. 14.

Step 2: integrate the BDD models for phases into the system BDD models by the PMS-BDD method. There are two kind of sort orders in the PMS-BDD, the backward PDO and the forward PDO. And through the backward PDO, the system BDD model is much smaller. By the backward PDO and taking the order $M 1_{4}<$ $M 1_{3}<M 1_{2}<M 1_{1}<M 2_{4}<M 2_{3}<M 2_{2}<M 2_{1}<M 3_{4}<M 3_{3}$ $<M 3_{2}<M 4_{2}<M 5_{3}$. Then, the system BDD models in the first two phases, in first three phases and in all four phases is shown in Fig. 15(a)-(c), respectively.

Step 3: according to the system BDD models shown in Fig. 15, we can get the disjoint paths for the ACOS in phase $1\left(\eta_{1}\right)$, in the first two phases $\left(\eta_{2}\right)$, in the first three phases $\left(\eta_{3}\right)$ and in all four phases are $\left(\eta_{4}\right)$,

$\left\{\begin{array}{l}\eta_{1}=M 1_{1} M 2_{1} \\ \eta_{2}=M 1_{2} M 2_{2} M 3_{2} M 4_{2} \\ \eta_{3}=M 1_{3} M 2_{3} M 3_{3} M 4_{2} M 5_{3} \\ \eta_{4}=M 1_{4} M 2_{4} M 3_{4} M 4_{2} M 5_{3}\end{array}\right.$

Step 4: integrate all the reliability indices of the module reliabilities and include the dynamic and static modules. As a result, the system reliability in different phases can be evaluated as, 


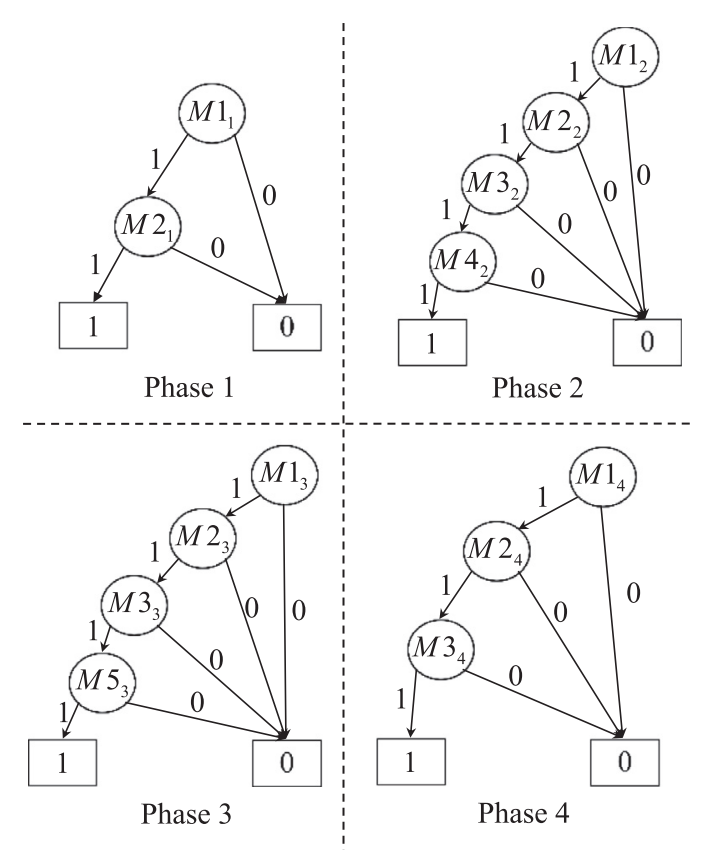

Fig. 14. The modularized BDD model for each phase of the AOCS.

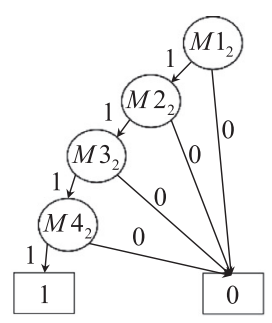

(a). First two Phase

(a) System BDD model for the first two phases.

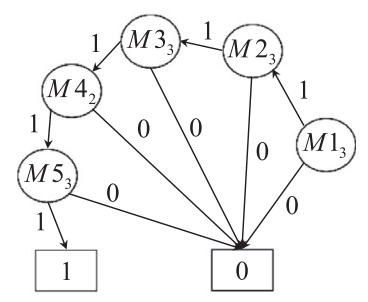

(b) System BDD model for the first three phases.

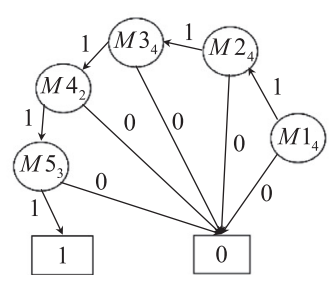

(c) System BDD model for the all the four phases.

Fig. 15. The system BDD models for different phases.

$$
\begin{aligned}
& R(t) \\
& \quad\left\{\begin{array}{cc}
\quad \leq T_{1} & 0<t \\
R_{M 1}(t) R_{M 2}(t), & T_{1} \\
R_{M 1}(t) R_{M 2}(t) R_{M 3}(t-T 1) R_{M 4}(t-T 1), & \\
<t \leq T_{1}+T_{2} & \\
R_{M 1}(t) R_{M 2}(t) R_{M 3}(t-T 1) R_{M 5}(t-(T 1+T 2)) R_{M 4}(T 2), & \\
T_{1}+T_{2}<t \leq T-T_{4} & \\
R_{M 1}(t) R_{M 2}(t) R_{M 3}(t-T 1) R_{M 5}(T 3) R_{M 4}(T 2), & \\
& T-T_{4}<t \leq T
\end{array}\right.
\end{aligned}
$$

where $T=\sum_{i=1}^{4} T_{i}$.

\subsection{Results}

\subsubsection{Comparison with the system without shocks}

The MC simulation of the system mission profile was performed with $2 \times 10^{5}$ histories in this paper. The system reliability of the phased AOCS considering random shocks is represented as the dashed line in Fig. 16. Moreover, the reliability of the same system without random shocks is represented as the solid line in Fig. 16. The reliabilities of the AOCS at the end of each phase are also shown in Table 2, as well as the relative difference between the reliabilities with and without random shocks.

As expected, when the AOCS travels a long time in the outer space, the system reliability is lower than that when considering random shocks, especially in phase 3 and phase 4 . If the random shocks are not considered in the modeling, the system reliability will be overestimated.

\subsubsection{Model confidence}

In this section, following the proposed MC simulation procedure, the reliability of the AOCS under infinite shocks and without shocks are evaluated. To assess the confidence of the estimated AOCS reliability under infinite random shocks, the system reliability evaluation by the MC simulation procedure are repeated for $N=200$ times and the results are shown in Table 3.

With the system reliabilities at different time shown in Table 3, the mean reliabilities values at different times are shown as the solid line in Fig. 17. The upper and lower bounds of the 95\% confidence of the reliability values at different times are represented as dashed lines in

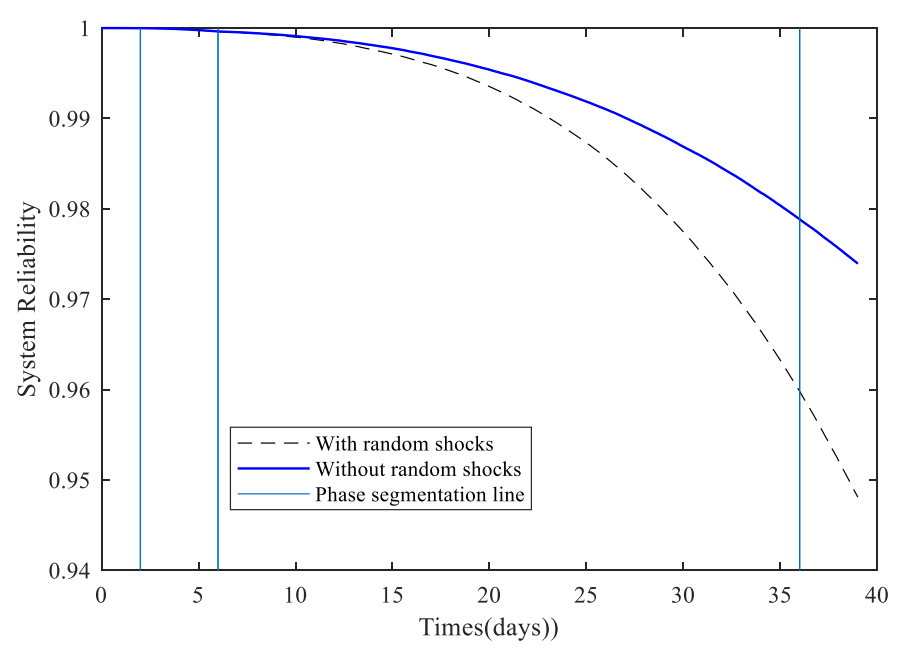

Fig. 16. The reliability of the AOCS with and without random shocks. 
Table 2

The results for the AOCS with and without random shocks.

\begin{tabular}{lllll}
\hline & Phase 1 & Phase 2 & Phase 3 & Phase 4 \\
\hline$R_{\text {sys }}^{\text {noshock }}(t)$ & 0.999990 & 0.999624 & 0.97885 & 0.973937 \\
$R_{\text {sys }}^{\text {shocks }}(t)$ & 0.999988 & 0.999613 & 0.95980 & 0.948101 \\
Relative difference & $1.5000-06$ & $1.1002 \mathrm{e}-05$ & 0.0198 & 0.0272
\end{tabular}

Table 3

The reliabilities for the AOCS under random shocks.

\begin{tabular}{llllll}
\hline Time (days) & 0 & 0.1 & 0.2 & $\ldots \ldots$ & 39 \\
\hline$R_{\text {sys }, 1}^{\text {shock }}$ & 1 & 1.000 & 1.000 & $\ldots \ldots$ & 0.9429 \\
$R_{\text {sys }, 2}^{\text {shocks }}$ & 1 & 1.000 & 1.000 & $\ldots \ldots$ & 0.9431 \\
$\ldots \ldots$ & $\ldots \ldots$ & $\ldots \ldots$ & $\ldots \ldots$ & $\ldots \ldots$ & $\ldots \ldots$ \\
$R_{\text {sys }, N=200}^{\text {shocks }}$ & 1 & 1.000 & 1.000 & $\ldots \ldots$ & 0.9426 \\
\hline
\end{tabular}

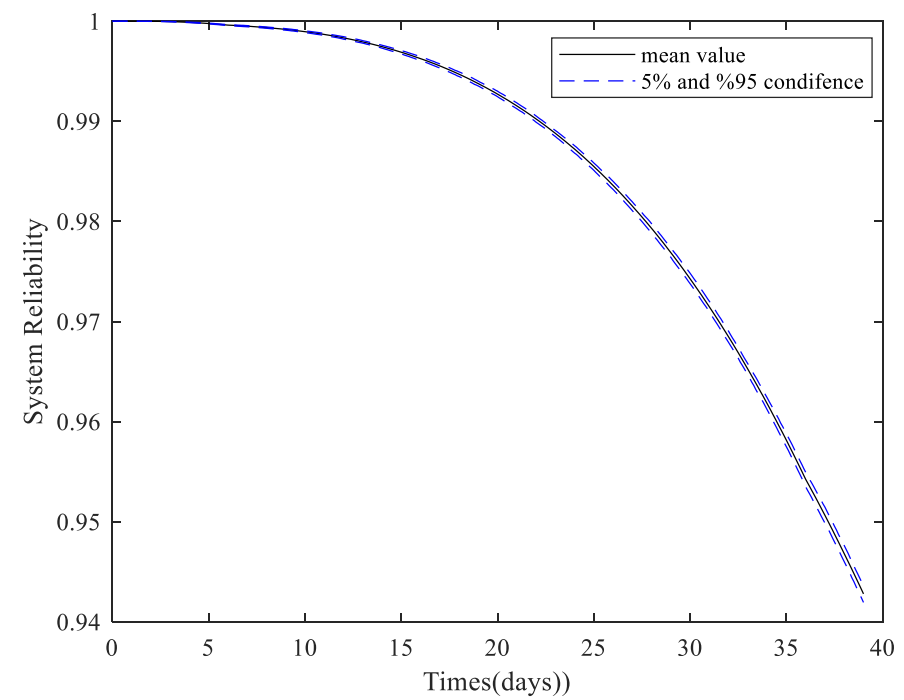

Fig. 17. The upper and lower bounds of the $95 \%$ confidence of the proposed MC method with $2 \times 10^{5}$ realizations.
Table 4

The errors of the results for the AOCS sensitivity analysis.

\begin{tabular}{llllllll}
$\begin{array}{l}\text { System reliability } \\
\text { error }\end{array}$ & 0.2 & 0.23 & 0.26 & 0.29 & 0.32 & 0.35 & 0.4 \\
\hline 0.247 & $0 \%$ & $0.45 \%$ & $0.98 \%$ & $1.46 \%$ & $1.93 \%$ & $2.42 \%$ & $3.05 \%$ \\
0.227 & $0.45 \%$ & $0.84 \%$ & $1.31 \%$ & $1.82 \%$ & $2.21 \%$ & $2.65 \%$ & $3.23 \%$ \\
0.212 & $0.87 \%$ & $1.27 \%$ & $1.66 \%$ & $2.10 \%$ & $2.55 \%$ & $2.85 \%$ & $3.39 \%$ \\
0.197 & $1.27 \%$ & $1.65 \%$ & $2.03 \%$ & $2.38 \%$ & $2.81 \%$ & $3.07 \%$ & $3.55 \%$ \\
0.182 & $1.65 \%$ & $2.02 \%$ & $2.35 \%$ & $2.66 \%$ & $3.04 \%$ & $3.25 \%$ & $3.68 \%$ \\
0.167 & $2.19 \%$ & $2.48 \%$ & $2.74 \%$ & $3.01 \%$ & $3.28 \%$ & $3.52 \%$ & $3.87 \%$ \\
\hline
\end{tabular}

Fig. 17, respectively. Consequently, it can be concluded that the proposed MC simulation method can provide accurate results with $2 \times 10^{5}$ realizations.

\subsection{Sensitivity analysis}

In this paper, the effect of random shocks to the PMS is studied. With respect to the random shocks modeling, we have analyzed the sensitivity of the system reliability estimates to two parameters which are related to the random shocks effect, the random shocks occurrence rate $u=[1 / 4,1 / 6]$ days $^{-1}$ and the relative increment in the transition rates $\varepsilon=[0.2,0.4]$. The estimated system reliabilities at the end of for different combinations of the two parameters are shown in Fig. 18.

According to the results in Fig. 18, it can be seen that with the increase of the relative increment $\varepsilon$ or the random shocks occurrence rate $u$, the system reliability decreases as expected. Higher $\varepsilon$ leads to larger components' failure rates, and larger occurrence rate $u$ values result in more random shocks over the whole lifetime, which also decreases the system reliability. In Table 4, the system reliability with parameters $\varepsilon=0.2$ and $u=1 /$ days $^{-1}$ is set to the standard and other elements are differences with different parameters combination. As shown in Table 4, when the same percentage of variation applies to two parameters, $\varepsilon$ is more influential than $u$ on the system reliability.

\section{Conclusions}

In this paper, an original reliability model of a PMS subjected to random shocks has been proposed together with a MC simulation procedure for its assessment. Dynamic behaviors, like the cold standby, and different lifetime distributions due to different component types are

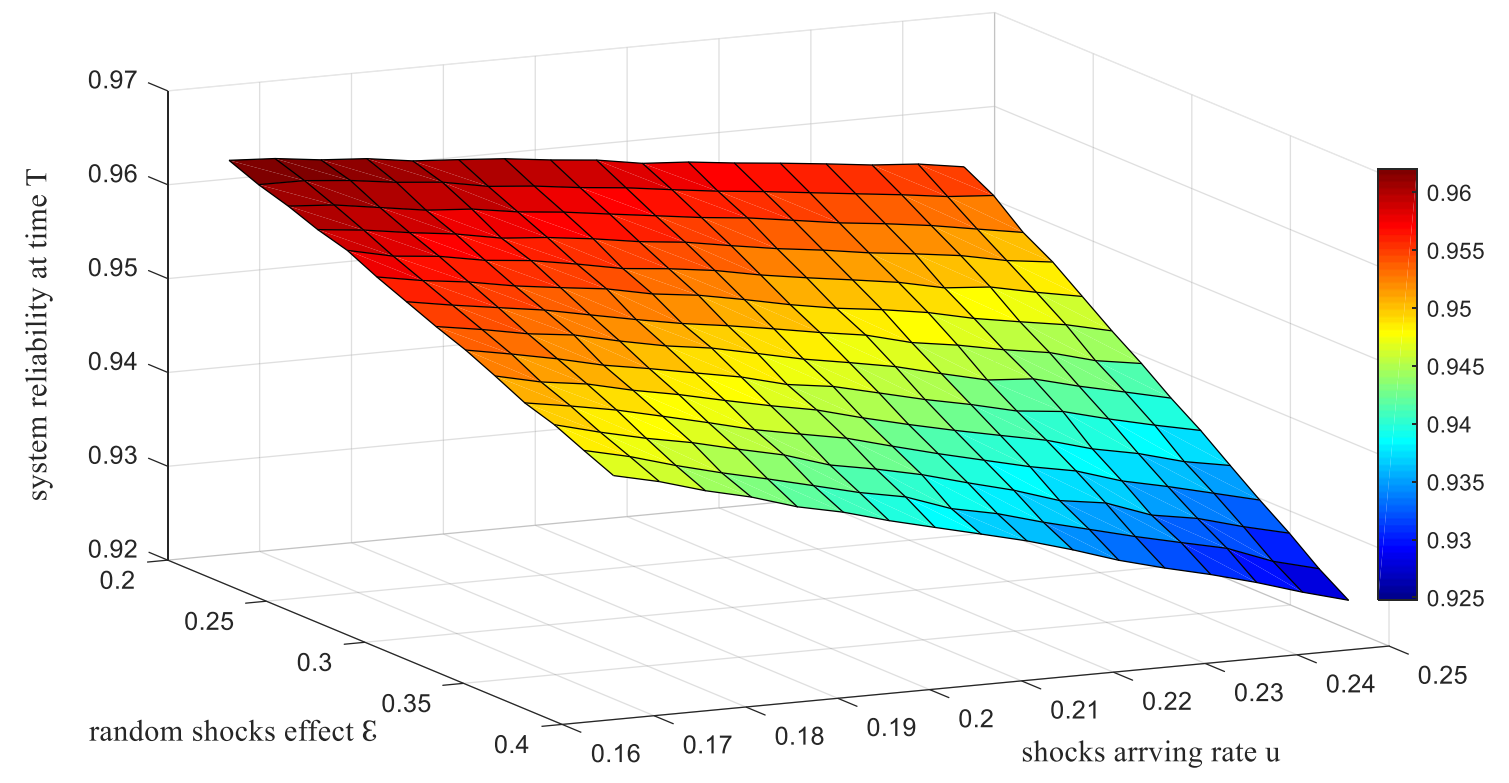

Fig. 18. The reliability of the phased AOCS with and without random shocks for different combinations of $u$ and $\varepsilon$. 
considered during the modeling.

A practical engineering case, the AOCS in the spacecraft, is used as a case study. To evaluate the system reliability under infinite random shocks effect, a Monte Carlo simulation procedure is proposed. The proposed MC simulation procedure is certified by a dynamic module under finite random shocks. The comparison of the reliability of the system considering the random shocks effect or not confirms the importance of the random shocks effects on the system reliability. At last, the sensitivity analysis involving the parameters that affect the random shocks effect is also carried out to characterize the influences of the random shocks model parameters.

In this paper, only the constant components' lifetime parameters provided by the designers are used to evaluate the reliability of PMS under random shocks. However, in reality, the uncertainties of these parameters have significant effects on the robustness of the system model as well as the system reliability, so this will be part of our future work. On the other hand, the shocks arriving rate may not always follow the homogeneous Poisson process: how to model and assess the system reliability under different random shocks arriving rate process will be another topic of our future research.

\section{Acknowledgments}

This study was sponsored by theNational Natural Science Foundation of China under Grant No. 51775090 and China Scholarship Council No.201606070066.

\section{References}

[1] Xing L. Reliability evaluation of phased-mission systems with imperfect fault coverage and common-cause failures. IEEE Trans Reliab 2007;56(1):58-68.

[2] Zang X, Sun N, Trivedi KS. A BDD-based algorithm for reliability analysis of phasedmission systems. IEEE Trans Reliab 1999;48(1):50-60.

[3] Xing L, Dugan JB. Analysis of generalized phased-mission system reliability, performance, and sensitivity. IEEE Trans Reliab 2002;51(2):199-211.

[4] Levitin G, Xing L, Amari SV. Recursive algorithm for reliability evaluation of nonrepairable phased mission systems with binary elements. IEEE Trans Reliab 2012;61(2):533-42.

[5] Xing L, Levitin G. BDD-based reliability evaluation of phased-mission systems with internal/external common-cause failures. Reliab Eng Syst Saf 2013;112:145-53.

[6] Tang Z, Dugan JB. BDD-based reliability analysis of phased-mission systems with multimode failures. IEEE Trans Reliab 2006;55(2):350-60.

[7] Mo Y, Xing L, Dugan JB. MDD-based method for efficient analysis on phased-mission systems with multimode failures. IEEE Trans Syst Man Cybern 2014;44(6):757-69.

[8] Alam M, Al-Saggaf UM. Quantitative reliability evaluation of repairable phasedmission systems using Markov approach. IEEE Trans Reliab 1986;35(5):498-503.

[9] Mura I, Bondavalli A. Markov regenerative stochastic Petri nets to model and evaluate phased mission systems dependability. IEEE Trans Comput 2001;50(12):1337-51.

[10] Wang C, Xing L, Levitin G. Competing failure analysis in phased-mission systems with functional dependence in one of phases. Reliab Eng Syst Saf 2012;108:90-9.

[11] Ou Y, Dugan J B. Modular solution of dynamic multi-phase systems. IEEE Trans
Reliab 2004;53(4):499-508.

[12] Meshkat L, Xing L, Donohue SK, et al. An overview of the phase-modular fault tree approach to phased mission system analysis. Proceedings of the international conference on space mission challenges for information technology. 2003.

[13] Golge S, O'Neill PM, Slaba TC. NASA galactic cosmic radiation environment model: Badhwar-O'Neill (2014). 34th international cosmic ray conference. 2015.

[14] Lin YH, Li YF, Zio E. Integrating random shocks into multi-state physics models of degradation processes for component reliability assessment. IEEE Trans Reliab 2015;64(1):154-66.

[15] Zheng B, Huang HZ, Guo W, et al. Fault diagnosis method based on supervised particle swarm optimization classification algorithm. Intell Data Anal 2018;22(1):191-210.

[16] Mi J, Li YF, Yang YJ, Peng W, Huang HZ. Reliability assessment of complex electromechanical systems under epistemic uncertainty. Reliab Eng Syst Saf 2016;152:1-15.

[17] Li W, Pham H. Reliability modeling of multi-state degraded systems with multicompeting failures and random shocks. IEEE Trans Reliab 2005;54(2):297-303.

[18] Rafiee K, Feng Q, Coit DW. Reliability modeling for dependent competing failure processes with changing degradation rate. IIE Trans 2014;46(5):483-96.

[19] Becker G, Camarinopoulos L, Kabranis D. Dynamic reliability under random shocks. Reliab Eng Syst Saf 2002;77(3):239-51.

[20] Ruiz-Castro JE. Markov counting and reward processes for analysing the performance of a complex system subject to random inspections. Reliab Eng Syst Saf 2016;145:155-68.

[21] Peng W, Li YF, Yang YJ, et al. Bayesian degradation analysis with inverse Gaussian process models under time-varying degradation rates. IEEE Trans Reliab 2017;66(1):84-96.

[22] Zheng B, Li YF, Huang HZ. Intelligent fault recognition strategy based on adaptive optimized multiple centers. Mech Syst Signal Process 2018;106:526-36.

[23] Peng W, Balakrishnan N, Huang HZ. Reliability modelling and assessment of a heterogeneously repaired system with partially relevant recurrence data. Appl Math Modell 2018;59:696-712.

[24] Guo J, Li YF, Zheng B, Huang HZ. Bayesian degradation assessment of CNC machine tools considering unit non-homogeneity. J Mech Sci Technol 2018;32(6):2479-85.

[25] Distefano S, Trivedi KS. Non-Markovian state-space models in dependability evaluation. Qual Reliab Eng Int 2013;29(2):225-39.

[26] Zio E, Pedroni N. Reliability estimation by advanced monte carlo simulation, simulation methods for reliability and availability of complex systems. London: Springer; 2010. p. 3-39.

[27] Zio E, Librizzi M. Direct Monte Carlo simulation for the reliability assessment of a space propulsion system phased mission. Proceedings of the 8th international conference on probabilistic safety assessment \& management (PSAM). ASME Press; 2006.

[28] Mi J, Li YF, Peng W, et al. Reliability analysis of complex multi-state system with common cause failure based on evidential networks. Reliab Eng Syst Saf 2018;174:71-81.

[29] Khoda T. Find modules in fault trees. IEEE Trans. Reliab 1989;38(2):165-76.

[30] Li YF, Mi J, Liu Y, et al. Dynamic fault tree analysis based on continuous-time Bayesian networks under fuzzy numbers. Proc Inst Mech Eng Part O 2015;229(6):530-41.

[31] Huang HZ, Huang CG, Peng Z, et al. Fatigue life prediction of fan blade using nominal stress method and cumulative fatigue damage theory. Int $\mathrm{J}$ Turbo JetEngines 2017https://doi.org/10.1515/tjj-2017-0015.

[32] Yang YJ, Peng W, Zhu SP, Huang HZ. A Bayesian approach for sealing reliability analysis considering the non-competing relationship of multiple degradation processes. Ekcsploatacja i Niezawodnosc Maint Reliab 2016;18(1):10-5.

[33] Li XY, Huang HZ, Li YF. Reliability analysis of phased mission system with nonexponential and partially repairable components. Reliab Eng Syst Saf 2018;175:119-27.

[34] Li XY, Huang HZ, Li YF, et al. Reliability assessment of multi-state phased mission system with non-repairable multi-state components. Appl Math Modell 2018;61:181-99. 